\title{
A semi-automated system for biofeedback-assisted relaxation therapy
}

\author{
ALAN T. POPE and CHARLES D. GERSTEN \\ FDR VA Hospital, Montrose, New York 10548
}

\begin{abstract}
A semi-automated system is described for use in research in biofeedback-assisted muscular relaxation therapy. The system employs a cassette tape recorder for presentation of instructions and for program control signals, programming modules for timing and gating EMG feedback and EMG recording intervals, a Kodak Carousel programmer to interface the cassette recorder with the programming system, and an EMG processor wired for external timing of feedback and counting of averaged EMG activity. A description of the current functioning of the system is presented, along with possible extensions.
\end{abstract}

Some of the more structured procedures used in behavior therapies, such as relaxation training and systematic desensitization, are readily automated to one degree or another. The instrumentation that has been employed ranges from a simply modified tape recorder (Migler \& Wolpe, 1967) to an on-line process control computer (Lang, 1969). The procedures have in common the use of pre-recorded audio tapes for the presentation of instructions and stimuli.

This paper describes a semi-automated system developed for research in biofeedback-assisted muscle relaxation therapy. The system represents the convergence of three developments: (a) the use of pre-recorded instructions, (b) the augmentation of natural cues arising from muscle tension by electromyographic (EMG) feedback, and (c) the automation of the presentation of the recorded material and physiological feedback and recording. The process control instrumentation employed is the programmable logic type, which provides some computer-like flexibility over hard-wired systems (Sidowski, 1975).

Progressive relaxation (Jacobson, 1938, 1970) is a therapeutic regime that consists of a substantial number of muscle exercises covering the major muscle groups of the body. Each exercise involves alternate tensing and relaxing of a particular muscle group. The entire training program has been used to promote deep muscular relaxation in tense individuals (Jacobson, 1938, 1970), but abbreviated versions involving fewer exercises and shorter periods of practice have been used extensively to promote the development of relaxation during systematic desensitization therapy (e.g., Wolpe, 1969). In its current usage, the present system employs a subset of the complete exercise series, although a substantially larger number of exercises can be accommodated easily.

A number of recent studies have shown that the augmentation of natural cues arising from muscle tension by means of EMG feedback can facilitate the reduction of muscle tension during relaxation training (e.g., Budzynski \& Stoyva, 1969; Budzynski, Stoyva \& Adler, 1970; Green, Walters, Green, \& Murphy, 1969).
In these studies, auditory (sometimes visual) stimuli, varying along a dimension such as tone frequency, track variations in muscle tension during the practice of relaxation. In the present system, the EMG recording and feedback apparatus developed by Budzynski and Stoyva (1969) and used in a number of EMG feedback studies (Budzynski, Stoyva, \& Adler, 1970; Budzynski, Stoyva, Adler, \& Mullaney, 1973; Raskin, Johnson, \& Rondestvedt, 1973; Wickramasekera, 1972) is used to provide feedback and to display averaged EMG activity in digital form.

A system to implement a biofeedback-assisted Jacobsonian training procedure is described in this paper. The system was designed to permit a wide variety of experimental manipulations including: (a) the number and the type of relaxation exercises, e.g., progressive relaxation (Jacobson, 1938, 1970), autogenic training (Luthe, 1969), cue-controlled relaxation (Russell \& Sipich, 1973), (b) parametric variations in the duration of intervals of activity (e.g., tensing, relaxing, rest period), (c) the temporal locus of feedback presentation within a sequence, e.g., during the tensing and the relaxing phase of a Jacobsonian exercise, during either phase alone, or during neither phase, and (d) different "mixes" of feedback scheduling, such as feedback presented on alternate repetitions of a particular exercise, on alternate exercises, and on altemate presentations of a set of exercises. Other possibilities not necessarily unique to this system include variations in between-mode (auditory or visual) and within-mode (e.g., binary, incremental, or proportional) feedback. These variations involve selection and arrangement of the pre-recorded materials, timer setting adjustments, and changes in a few plug-in connections.

\section{SYSTEM FUNCTION FOR RELAXATION TRAINING}

In this application, the system functions to instruct the subject in the Jacobsonian exercises and to record EMG activity and provide EMG biofeedback while each exercise is being carried out. The time line diagram of 


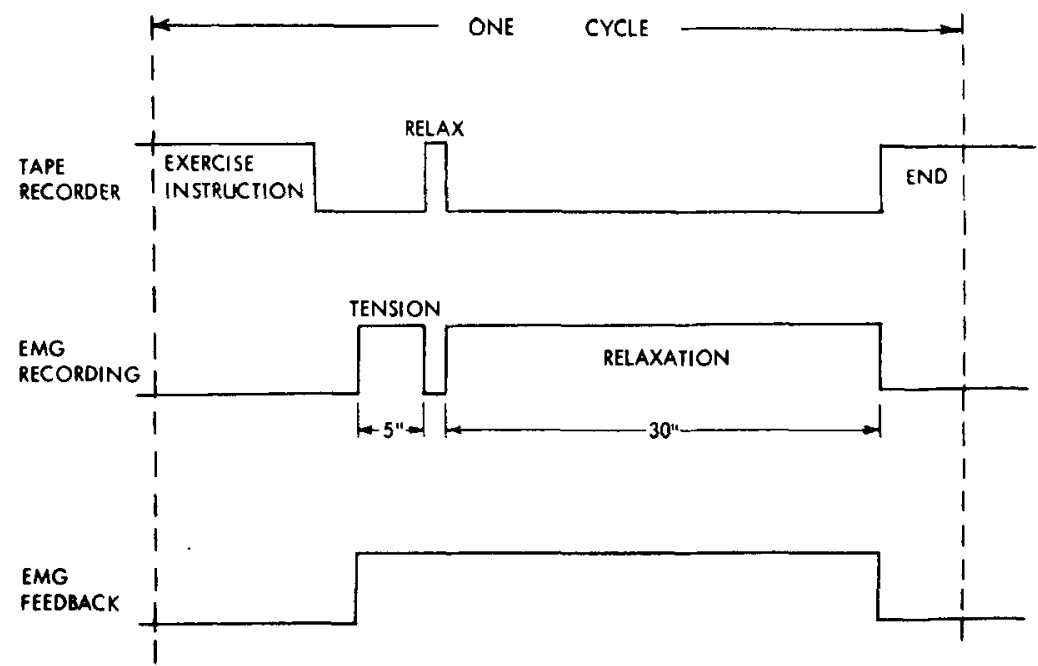

Figure 1. The sequence of events in one cycle of operation.
Figure 1 illustrates the temporal relationships of these operations. The following automatic sequence of events is initiated by a single button press by the experimenter, indicating that the subject is following the exercise instructions properly: (a) an EMG recording in terval of adjustable duration (currently $5 \mathrm{sec}$ ) during which the subject is tensing the prescribed muscle group, (b) a tape-recorded message to "relax," (c) another adjustable recording interval (currently $30 \mathrm{sec}$ ), and (d) a taped message terminating the exercise and giving the next exercise instruction. The program then shuts down until the initiation of the next cycle by the experimenter.
Auditory and/or visual feedback can be made available during any combination of the recording and message in tervals.

\section{SYSTEM COMPONENTS}

The components of the system and the function of each will be described with reference to the block diagram of Figure 2. The system employs an Audiotronics cassette tape recorder for presenting the instructions for the Jacobsonian relaxation exercises. Program control signals $(6.5 \cdot \mathrm{kHz}$ pulses $)$ are also

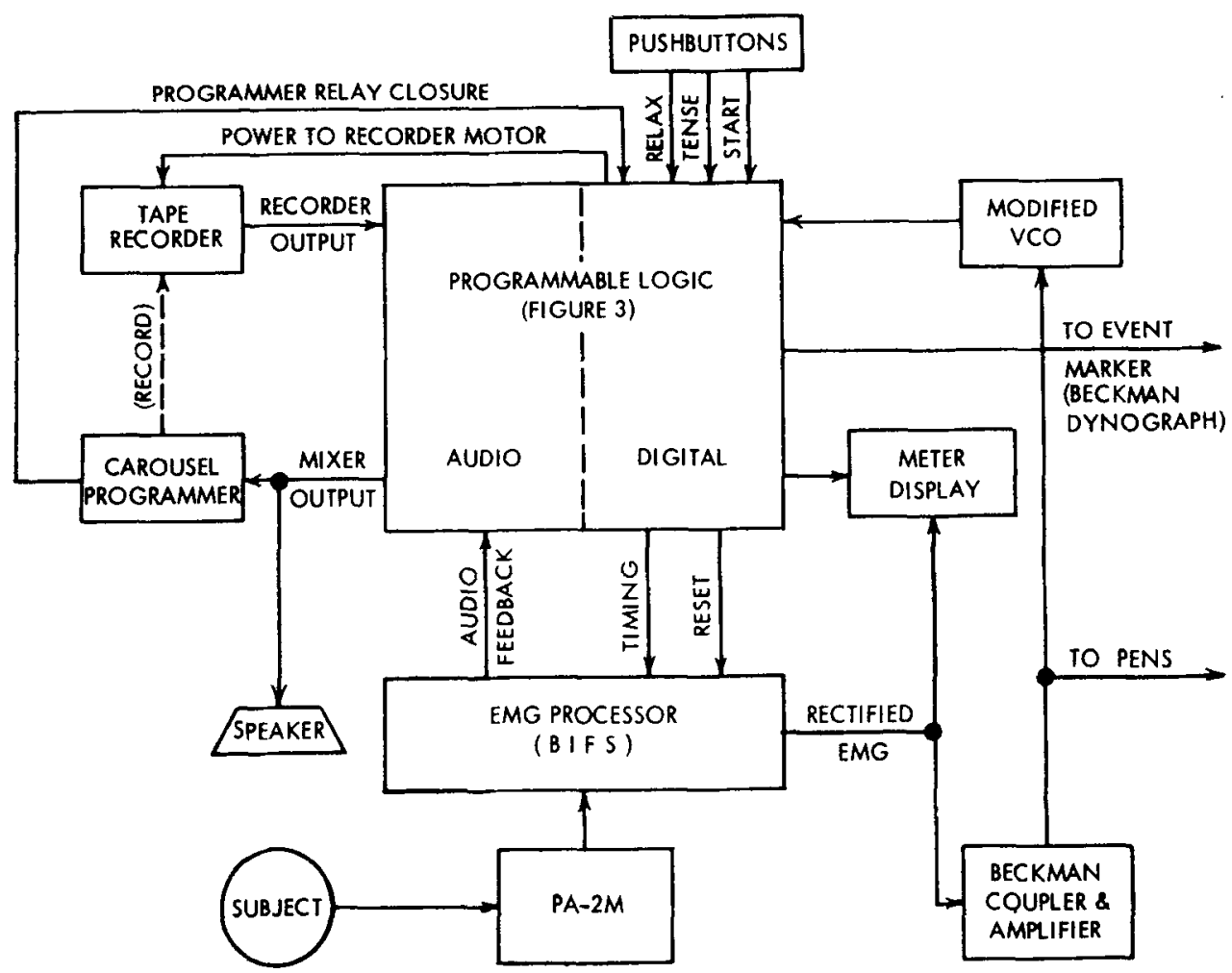

Figure 2. Block diagram of control interconnections of the major components of the system. 
pre-recorded on the instruction tape. The tape recorder is rewired so that power is supplied to the recorder electronics all of the time system power is on, but power to the drive motor is under programmed control. This allows the tape recorder to be paused during appropriate intervals in the automatic sequence.

The program control signals are pre-recorded on the instruction tape using a Kodak Carousel programmer. During recording, the programmer functions as a $6.5-\mathrm{kHz}$ oscillator and mixer that channels the manually pulsed signal and the voice instructions into the recorder input. A control signal is recorded after each exercise description and after each "relax" message. During playback, the programmer functions as a narrow-band frequency selective amplifier and relay driver, tuned to $6.5 \mathrm{kHz}$. Thus, a relay contact closure signals the end of each instruction and of each "relax" message to the logic control system.

The Bio-Feedback Systems (BFS) Model B-1 bioelectric information feedback system (BIFS) is the electromyographic signal processing unit in the system. The microvolt-level EMG activity at the muscle site (usually frontalis) is picked up by surface electrodes and amplified and filtered before being fed into the BIFS. The BIFS provides a digital display of the average EMG level for a preselected time interval. The BIFS also produces an audio tone that varies in frequency with momentary variations in the level of EMG activity. In the present system, several of the internal BIFS functions have been brought out for external manipulation and control. The timing and reset functions of the digital display were wired for control by the programmable logic system. The rectified and smoothed EMG signal was brought out to drive various devices described below. Finally, the audio feedback signal was routed through the logic system for gating into an external speaker.

The rectified EMG signal is routed to drive three different devices. The signal is amplified to drive a visual feedback display. The visual display consists of a meter specially constructed so that its scale and moving needle may be projected with a filmstrip projector for an enlarged feedback display.

The EMG signal is also fed into a Beckman Type RM dynograph recorder to obtain a polygraph record of momentary changes in EMG level. The auxiliary output of the dynograph amplifier is routed to a voltage-controlled oscillator (VCO), modified so that the output pulse rate is in a slower range. The VCO output is utilized by the logic system to provide a compound type of auditory feedback. That is, as EMG level rises, not only does the frequency of the feedback tone increase, but the rate at which the tone pulses on and off also increases.

\section{LOGIC CONTROL SYSTEM}

The programmable digital logic system (Coulbourn Instruments) is the heart of the present system, and its sequence of operation in interaction with the devices previously described will be outlined with reference to the system block diagram in Figure 2 and the logic diagram of Figure 3.

A session is started by pressing the "START" pushbutton to reset all flip-flops except Flip-Flop 3 (FF 3), which is set to provide power to the tape drive motor. FF 2 may be prewired to enable feedback to be presented during the appropriate intervals of every other exercise (intermittent schedule), beginning on either the first or second exercise; or its output may be disconnected to enable continuous feedback. The addition of a predetermining counter extends the variations of intermittent feedback.

During the baseline measurement period, average EMG levels for 30 -sec trials are obtained from the BIFS digital display by operating the $30-\mathrm{sec}$ timer by depressing the "RELAX" button. The end pulse (EP) of the "relax" timer is blocked from having an effect on the system by the operation of FF 1 and And Gate 3 (A 3).

The first exercise instruction is presented by activating the tape recorder with its "PLAY" key. For initial sessions, the tape begins with introductory instructions, followed by the first exercise instruction. The signal following the instruction resets FF 3 , shutting off the recorder, and the system is quiescent. The one-shot output of FF 3 is blocked from further effect at this time by the operation of FF 4 and A 4 . However, the operation of the "TENSE" button is enabled by FF 3 and A 1. The inputs to A 1 serve to disable this button when the recorder or the relaxation timer are functioning, in order to prevent accidental triggering of the automatic sequence.

The automatic sequence of operation is initiated when the experimenter observes that the subject is correctly following the exercise instruction and presses the "TENSE" button. The 5 -sec timer is wired so that it may be restarted repeatedly within the timing interval, if necessary. The button press resets the BIFS digital display to zero and begins the counting of EMG activity during the "tension" interval.

The button press also sets FF 4 to enable the presentation of the compound auditory feedback described earlier. As it is diagrammed in Figure 3, the system is wired to present feedback until the end of the relaxation interval. However, feedback may be presented during any combination of the timing intervals by wiring the appropriate timing (T) output into Or Gate 4(O 4).

When the $5-\mathrm{sec}$ tension interval has elapsed, the tape recorder is actuated, and a brief message to relax is presented. The taped control signal following the message resets FF 3 , shutting off the tape recorder. However, this time the one-shot output of FF 3 is enabled by FF 4 and A 4 to operate the $30 \mathrm{sec}$ timer, reset the digital display, and record EMG activity for the relaxation interval. The end pulse (EP) output of the timer is enabled by FF 1 and A 3 to change the state of the intermittent feedback FF 2 (is used), to tum off 


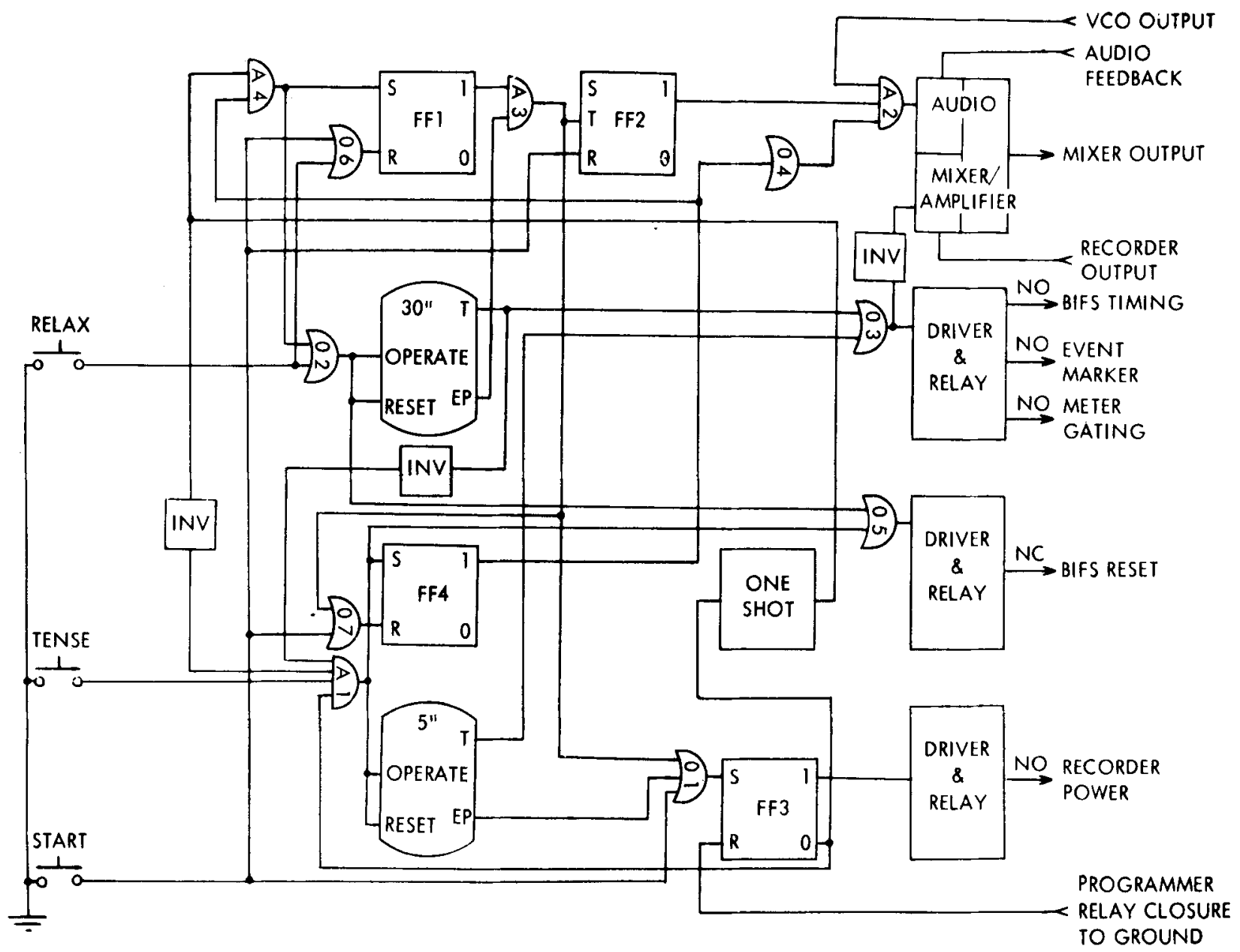

Figure 3. Circuitry of the programmable logic system indicated in Figure 2.

feedback by means of FF 4, and to actuate the tape recorder. A message is presented, terminating the exercise and indicating that the subject may shift around. After a blank section on the tape, the next exercise instructions are given. The signal following the instructions shuts down the system until the next cycle is initiated by the experimenter's button press.

Permanent recording of the EMG digital display at the end of each tension and each relaxation interval is now done manually by the experimenter. This function could be performed by a printing counter, thus freeing the experimenter to perform other functions, if desired. The presence of the experimenter in the treatment room can be obviated by initiating each cycle from a physiological parameter or a switch closure by the subject.

The tension and relaxation interval durations are recorded concurrently with the momentary EMG level by an event marker on the Beckman polygraph.

The time line diagram of Figure 1 is readily translated into a state diagram utilizing a state notational language developed for describing behavioral procedures and data acquisition formats (Snapper \& Kadden, 1973). The procedure could then be implemented on a minicomputer, utilizing any one of a number of automatic sequence control languages, e.g., SKED (Kadden, 1974). However, one would probably want to take advantage of the greater capability of the minicomputer to implement interactive procedures in which the verbal material and/or feedback to a subject depends upon the subject's performance or progress (Castellan, 1975).

\section{REFERENCES}

Budzynski, T. H., \& Stoyva, J. M. An instrument for producing deep muscle relaxation by means of analog information teedback. Joumal of Applied Behavior Analysis, 1969, 2. 231-237.

Budzynski, T. H., Stoyva, J. M., \& Adler, C. S. Feedbackinduced muscle relaxation: Application to tension headache. Journal of Behavior Therapy and Experimental Psychiatry, 1970, 1, 205-211.

Budzynski. T. H., Stoyva, J. M., Adler, C. S., \& Mullaney, C. M. EMG biofeedback and tension head ache: A controlledoutcome study. Psychosomatic Medicine, 1973, 35, 484-496.

CAstellan, N. John, Jr. The modern mini-computer in laboratory automation. American Psychologist, 1975, 30, 205-211. 
Green, E., Walters, E., Green, A., \& Murphy, G. Feedback technique for deep relaxation. Psychophysiology, 1969, 6. 371-377.

JACOBSON, E. Progressive relaxation. Chicago: University of Chicago Press, 1938.

JaCoBson, E. Modern treatment of tense patients. Springfield, 11l: Charles C Thomas, 1970.

Kanden, R. M. State notation and SKED: A general system for control and recording of behavioral experiments. Behavior Research Methods \& Instrumentation. 1974, 6. $167-170$.

LANG, P. J. The on-line computer in behavior therapy research. American Psychologist, 1969. 24. 236-239.

LuThe, W. (Ed.), Autogenic therapy (5 volumes). New York: Grune \& Stratton. 1969.

Migler, B., \& Wolpe, J. Automated self-desensitization: A case report. Behavior Research and Therapy. 1967, 5. 133-135.

Raskin, M., Johnson, G., \& Rondestvedt. J. W. Chronic anxiety treated by feedback-induced muscle relaxation. Archives of General Psychiatry, 1973, 28, 263-367.
Russell, R. K., \& SiPICh, J. F. Cue-controlled relaxation in the treatment of test anxiety. Journal of Behavior Therapy and Experimental Psychiatry, 1973, 4, 47-49.

SIDOWSKI, J. B. Instrumentation and computer technology: Applications and intluences in modern psychology. American Psychologist, 1975, 30, 191-196.

SNAPPER, A. G.. \& KADDEN, R. M. Time-sharing in a small computer based on a behavioral notation system. In B. Weiss (Ed.). Digital computers in the behavioral laboratory. New York: Applcton-Century-Crofts. 1973.

WICKRAMASEKERA, I. Instructions and EMG feedback in systematic desensitization: A case report. Behavior Therapy, 1972. 3, 460-465.

WOLPE, J. The practice of behavior therapy. New York: Pergamon Press. 1969.

(Received for publication March 21, 1975; revision received May 12, 1975.) 